\title{
HYPERSPACES OF HEREDITARILY INDECOMPOSABLE PLANE CONTINUA
}

\author{
E. D. TYMCHATYN ${ }^{1}$
}

\begin{abstract}
In this note we prove that if $X$ is a hereditarily indecomposable plane continuum then the hyperspace $C(X)$ can be embedded in Euclidean 3-space.
\end{abstract}

If $X$ is a continuum, we let $C(X)$ denote the hyperspace of subcontinua of $X$ with the Hausdorff metric. A continuous function $\mu$ from $C(X)$ into the reals $R$ is called a Whitney map if the following two conditions are satisfied:

(i) $A \subset B$ and $A \neq B \Rightarrow \mu(A)<\mu(B)$.

(ii) $\mu(\{x\})=0$ for each $x \in X$.

Whitney maps always exist [4].

The aim of this note is to prove the following:

THEOREM. If $X$ is a hereditarily indecomposable plane continuum, then $C(X)$ is embeddable in Euclidean 3-space $R^{3}$.

Transue [3] proved this theorem for the case in which $X$ is a continuum which does not separate the plane. Krasinkiewicz [2] proved that $C(X)$ is embeddable in $R^{4}$. J. T. Rogers recently announced the theorem in case $X$ separates the plane into finitely many disjoint regions. I am grateful to Professor Krasinkiewicz for pointing out this problem to me. The argument that is given here is a modification of that given by Transue [3].

Proof. We suppose without loss of generality that $X$ is contained in the 2sphere $S^{2}$. Let $p \in X$. Let $P$ denote the set of continua in $X$ which contain $p$. We prove that $P$ is an arc in $C(X)$.

It is clear that $P$ is compact. If $P$ is not connected then $P$ can be written as the union of two mutually separated sets $H$ and $K$. We may suppose $X \in H$. Since $K$ is compact there is a maximal element $T$ of $K$ (i.e. $T$ is contained in no other element of $K$ ). Then $T$ is in the closure of $H$ for if $U$ is any neighborhood of $T$ in $X$, then the closure of the component of $U$ which contains $T$ is an element of $P$ which properly contains $T$ and hence is in $H$. This is a contradiction since $H$ and $K$ are separated sets. We have proved that $P$ is a continuum.

Received by the editors June 30, 1975 and, in revised form, October 6, 1975.

AMS (MOS) subject classifications (1970). Primary 54B20, 54F50, 54F20.

Key words and phrases. Hyperspaces, planar, hereditarily indecomposable, continua, embedding in $E^{3}$.

1 This research was supported in part by a grant from the National Research Council of Canada. 
If $S, T \in P$ then either $S \subset T$ or $T \subset S$ since $X$ is hereditarily indecomposable and $p \in S \cap T$. Thus, $P$ is totally ordered under inclusion.

Let $\mu: C(X) \rightarrow R$ be a Whitney map. It follows from the above argument that $\left.\mu\right|_{P}$ carries $P$ homeomorphically onto the interval $[0, \varepsilon]$ where $\varepsilon=\mu(X)$.

Let $\sim$ be the equivalence relation defined on $X \times[0, \varepsilon]$ whose equivalence classes are the sets $g \times\{\mu(g)\}$ for $g \in C(X)$. To see that $\sim$ is upper semicontinuous, let $\left.\left(x_{i}, a_{i}\right)\right)$ and $\left.\left(y_{i}, a_{i}\right)\right)$ be sequences in $X \times[0, \varepsilon]$ such that for each $i,\left(x_{i}, a_{i}\right) \sim\left(y_{i}, a_{i}\right)$ and $\lim x_{i}=x, \lim y_{i}=y$ and $\lim a_{i}=a$. For each $i$ there exists $g_{i} \in C(X)$ such that $\mu\left(g_{i}\right)=a_{i}$ and $x_{i}, y_{i} \in g_{i}$. Without loss of generality we may suppose the sequence $g_{i}$ ) converges to $g$ in the compact space $C(X)$. Since $\mu$ is continuous $\mu(g)=\lim \mu\left(g_{i}\right)=\lim a_{i}=a$. Clearly, $x, y \in g$ so $(x, a) \sim(y, a)$ and $\sim$ is upper semicontinuous.

Let $U_{1}, U_{2}, \ldots$ be the complementary components of $X$ in $S^{2}$. For each $k \in C(X)$ let $k^{*}=k \cup \cup\left\{U_{i} \mid \mathrm{Bd}\left(U_{i}\right) \subset k\right\}$ where $\mathrm{Bd}\left(U_{i}\right)$ denotes the boundary of $U_{i}$.

We show that

$$
k^{*}=k \cup \cup\left\{W \mid W \text { is a component of } S^{2} \backslash k \text { and } W \subset S^{2} \backslash X\right\} .
$$

If $W$ is a component of $S^{2} \backslash k$ then $\operatorname{Bd}(W) \subset k$ since $k$ is closed and $W$ is open (since $S^{2}$ is locally connected). If $W \subset S^{2} \backslash X$ then $W$ is a component of $S^{2} \backslash X$ and so $W \subset k^{*}$. Let $U_{i} \subset k^{*}$. Then $\operatorname{Bd}\left(U_{i}\right) \subset k$. Now, $U_{i}$ is a component of $S^{2} \backslash X$. Since $\operatorname{Bd}\left(U_{i}\right) \subset k, U_{i}$ is also a component of $S^{2} \backslash k$. This completes the proof of $(\dagger)$.

Since $X$ is indecomposable, $X \backslash k$ is connected. It follows that at most one component of $S^{2} \backslash k$ meets $X$ and so $S^{2} \backslash k^{*}$ is connected and open. Thus, $k^{*}$ is closed. It is clear from the definition of $k^{*}$ that $k^{*}$ is connected. We have proved that $k^{*}$ is a continuum which does not separate $S^{2}$.

We extend $\sim$ to an equivalence relation $\sim^{*}$ on $S^{2} \times[0, \varepsilon]$ where the equivalence classes of $\sim^{*}$ are points and the sets $g^{*} \times\{\mu(g)\}$ where $g$ $\in C(X)$. For each $a \in[0, \varepsilon]$ the members of $\mu^{-1}(a)$ are pairwise disjoint so the sets $g^{*} \times\{\mu(g)\}$ are also pairwise disjoint by the definition of $g^{*}$. It remains to prove that $\sim^{*}$ is upper semicontinuous.

Let $\left.\left(x_{i}, a_{i}\right)\right)$ and $\left.\left(y_{i}, a_{i}\right)\right)$ be sequences in $S^{2} \times[0, \varepsilon]$ such that $\left.\left(x_{i}, a_{i}\right)\right)$ converges to $\left.(x, a),\left(y_{i}, a_{i}\right)\right)$ converges to $(y, a)$ and for each $i,\left(x_{i}, a_{i}\right)$ $\sim^{*}\left(y_{i}, a_{i}\right)$. If $x_{i}=y_{i}$ for infinitely many $i$, then $x=y$ and $(x, a) \sim^{*}(y, a)$. We suppose, therefore, that for each $i, x_{i} \neq y_{i}$. For each $i$ let $g_{i} \in C(X)$ such that $x_{i}, y_{i} \in g_{i}^{*}$ and $\mu\left(g_{i}\right)=a_{i}$. We may also suppose that the sequence $\left.g_{i}\right)$ converges to $g$ in $C(X)$. Since $\mu$ is continuous, $\mu(g)=\lim \mu\left(g_{i}\right)=\lim a_{i}=a$. If for each $i, x_{i} \in X$, then $x_{i} \in g_{i}$ and $x \in g$. Let us suppose now that for each $i, x_{i} \notin X$. For each $i$, let $U_{j_{i}}$ be the component of $S^{2} \backslash X$ which contains $x_{i}$. Then Bd $\left(U_{j_{i}}\right) \subset g_{i}$. If $x \in X$ then either $x \in g_{i}$ or $g_{i}$ separates $x$ and $x_{i}$ in $S^{2}$. In either case $x \in \lim g_{i}=g$. If $x \in U_{k}$ for some $k$ then for all sufficiently large $i, x_{i} \in U_{k}$ and $U_{j_{i}}=U_{k}$. Hence $\mathrm{Bd}\left(U_{j_{i}}\right)=\mathrm{Bd}\left(U_{k}\right) \subset g_{i}$ for all sufficiently large $i$. In particular $\mathrm{Bd}\left(U_{k}\right)=\lim \mathrm{Bd}\left(U_{j_{i}}\right) \subset \lim g_{i}=g$. In all cases $x \in g^{*}$. Similarly, $y \in g^{*}$ and so $(x, a) \sim^{*}(y, a)$. This completes the proof that $\sim^{*}$ is upper semicontinuous.

Let $\pi$ be the natural projection of $S^{2} \times[0, \varepsilon]$ onto the quotient space 
$\left(S^{2} \times[0, \varepsilon]\right) / \sim^{*}$. Let $h:\left(S^{2} \times[0, \varepsilon]\right) / \sim^{*} \rightarrow[0, \varepsilon]$ be such that $h(\pi(x, a))=a$. Then $h$ is 0 -regular as in the proof of Theorem 8 in [1]. By Moore's theorem $h^{-1}(\delta)$ is a 2 -sphere for each $\delta$ such that $0 \leqslant \delta<\varepsilon$ and $h^{-1}(\varepsilon)$ is a point. It follows from Theorem 7 in [1] that $\left(S^{2} \times[0, \varepsilon]\right) / \sim^{*}$ is a 3 -cell. This completes the proof of the theorem for $(X \times[0, \varepsilon]) / \sim$ is homeomorphic to $C(X)$ and $(X \times[0, \varepsilon]) / \sim$ is embedded in the 3 -cell $\left(S^{2} \times[0, \varepsilon]\right) / \sim^{*}$.

\section{REFERENCES}

1. E. Dyer and M. E. Hamstrom, Completely regular mappings, Fund. Math. 45 (1958), 103-118. MR 19, 1187.

2. J. Krasinkiewicz, On the hyperspaces of certain plane continua (to appear).

3. W. R. R. Transue, On the hyperspace of subcontinua of the pseudoarc, Proc. Amer. Math. Soc. 18 (1967), 1074-1075. MR 36 \#5901.

4. H. Whitney, Regular families of curves, Proc. Nat. Acad. Sci. U.S.A. 18 (1932), 275-278.

5. G. T. Whyburn, Analytic topology, Amer. Math. Soc. Colloq. Publ., vol. 28, Amer. Math. Soc., Providence, R. I., 1942. MR 4, 86.

6. J. L. Kelley, Hyperspaces of a continuum, Trans. Amer. Math. Soc. 52 (1942), 22-36. MR 3, 315 .

Department of Mathematics, University of Saskatchewan, Saskatoon, Canada

Department of Mathematics, University of Oregon, Eugene, Oregon 97403 